\title{
Reasoning through the Gut: The Microbiota-Gut-Brain Axis and its Role in the Pathogenesis of Autism Spectrum Disorder
}

\author{
Shreyas Singh ${ }^{1}$ and Avan Erhunmwunsee Dalton ${ }^{2+}$
}

${ }^{1}$ Skyline High School, Sammamish, WA, USA

${ }^{2+}$ Department of Biomedical Engineering, College of Medicine, University of Lagos, Nigeria

+Advisor

DOI: https://doi.org/10.47611/jsrhs.v10i2.1771

\section{ABSTRACT}

The age-old phrase 'gut feeling' is increasingly finding a scientific basis. The human gut houses a massive colony of microorganisms that not only maintain intestinal function, but also have far-reaching connections to the brain through a complicated 'microbiota-gut-brain' (MGB) axis. This axis has now been established to have implications in the management of various neuro-psychological conditions including Autism spectrum disorder (ASD), a condition that affects the way people communicate, behave and interact with others. Once considered a rare disorder, it is now estimated to have a prevalence of about 1 in 54 children in the United States. It is unfortunate that the existing cognitive-behavioral therapy protocols for ASD are prolonged and expensive, and that there is no currently approved medication for the core symptoms of the disorder. Scientists are now exploring the MGB axis for establishing possible therapeutic targets to alleviate the symptoms of ASD. This review attempts to highlight how the MGB axis works, and to capture the essence of the mechanisms that implicate the MGB axis in the pathogenesis of ASD. Further research in this direction may convincingly establish the role of novel treatment options for ASD ranging from probiotics and dietary modifications to newer modalities like fecal transplants, vagal nerve stimulation and gene therapy. Microbes, and not just behavioral intervention therapy, may hold the key to treating ASD.

\section{Introduction}

The human gastrointestinal (GI) tract contains a large colony of microorganisms collectively known as the gut 'microbiota'. It includes a host of viruses, bacteria, protozoa, fungi, and archaea in astronomical numbers. The number of bacteria alone in this microbial colony has been estimated to be approximately $3.8 \times 10^{13}$ - a figure that is even larger than the total number of human cells (Sender et al. 2016). Over the last decade, there has been growing interest in evaluating how the gut microbiota affects the 'gut-brain axis'- a bidirectional interaction between the gut and the brain. There is increasing evidence that the gut microbiota influence a complex interplay between the humoral, neural, immune, and endocrine systems, all of which link the peripheral intestinal system to the cognitive and emotional centers of the brain (Crumeyrolle-Arias et al. 2014).

The importance of the microbiota-gut-brain axis in mental and psychological health is underlined by reports of various GI tract symptoms occurring in patients with neurological and psychological disorders. Major depressive disorders are reportedly associated with loss of appetite and weight changes (Privitera et al, 2013). Patients with anxiety disorders (Mussell et al. 2008), schizophrenia (Severance et al. 2015) and Parkinson's disease (Edwards et al. 1993) commonly report GI tract symptoms like nausea and diarrhea. Mood disturbances, anxiety and stress have been recognized to play a role in GI tract disorders such as irritable bowel syndrome, inflammatory bowel disease (Graff et al. 2009) 
and peptic ulceration (Lim et al. 2014). The focus of the current study was to review the impact of the gut microbiota on the development of Autism spectrum disorder (ASD), a neuro-developmental disorder characterized by impairments in behavior, social and communication skills.

\section{Methodology}

This study aimed to review the functioning of the microbiota-gut-brain axis and the mechanisms by which the gut microbiota influence the pathogenesis of ASD. Original articles and meta-analyses for the review were selected by means of an internet search in PubMed, ScienceDirect, Hindawi and Google Scholar. The search criteria included the terms 'gut-brain axis', 'gut-brain communication', 'microbiota and mental health', 'microbiota and autism', 'gut microbiota and ASD'. Manuscripts written in English alone were included in this review.

\section{Discussion}

\section{Enteric nervous system}

The enteric nervous system (ENS) is one of the three divisions of the autonomic nervous system (Langley 2010), the others being the sympathetic and the parasympathetic systems. The ENS forms a large division of the peripheral nervous system (PNS) (Figure1) that regulates the functioning of the GI tract independent of the central nervous system (CNS) input. Known as the "second" brain of the human body, it consists of an intricate network of more than 100 million neurons in the gut (Furness 2006).

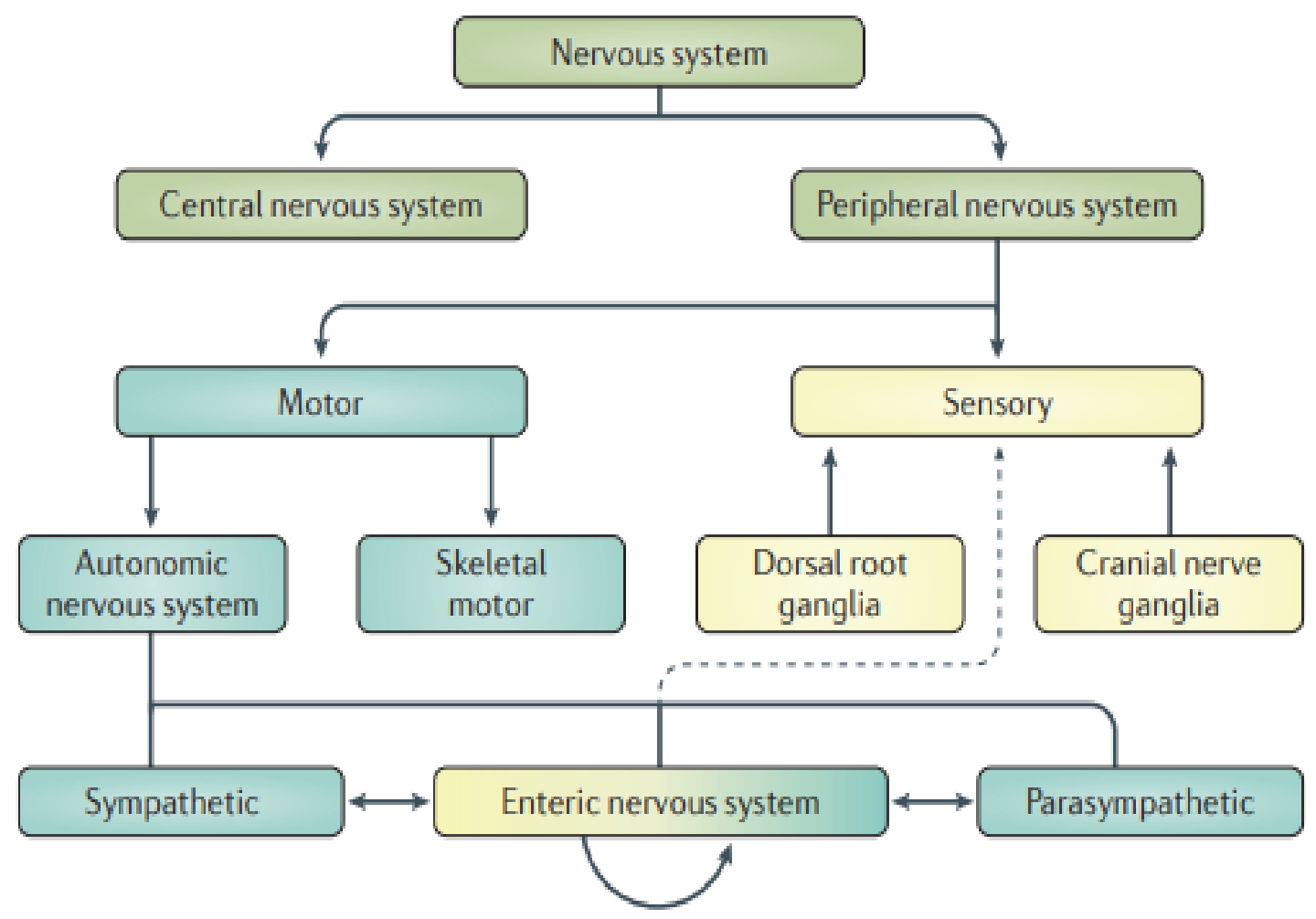


Figure 1. Interconnections between the ENS and components of the peripheral nervous system (Reproduced from Rao and Gershon 2016).

The neural control mechanism of the gut is highly complex (Figure 2), unlike the rest of the PNS. The ENS is adapted for integrative neuronal activity and independent control of the GI tract without the need for signals from the brain or spinal cord (Furness et al. 2014). Since the ENS and CNS share common neurotransmitters, signal pathways and anatomical characteristics, the pathophysiological mechanisms underlying CNS disease are often associated with GI symptoms (Figure 2).

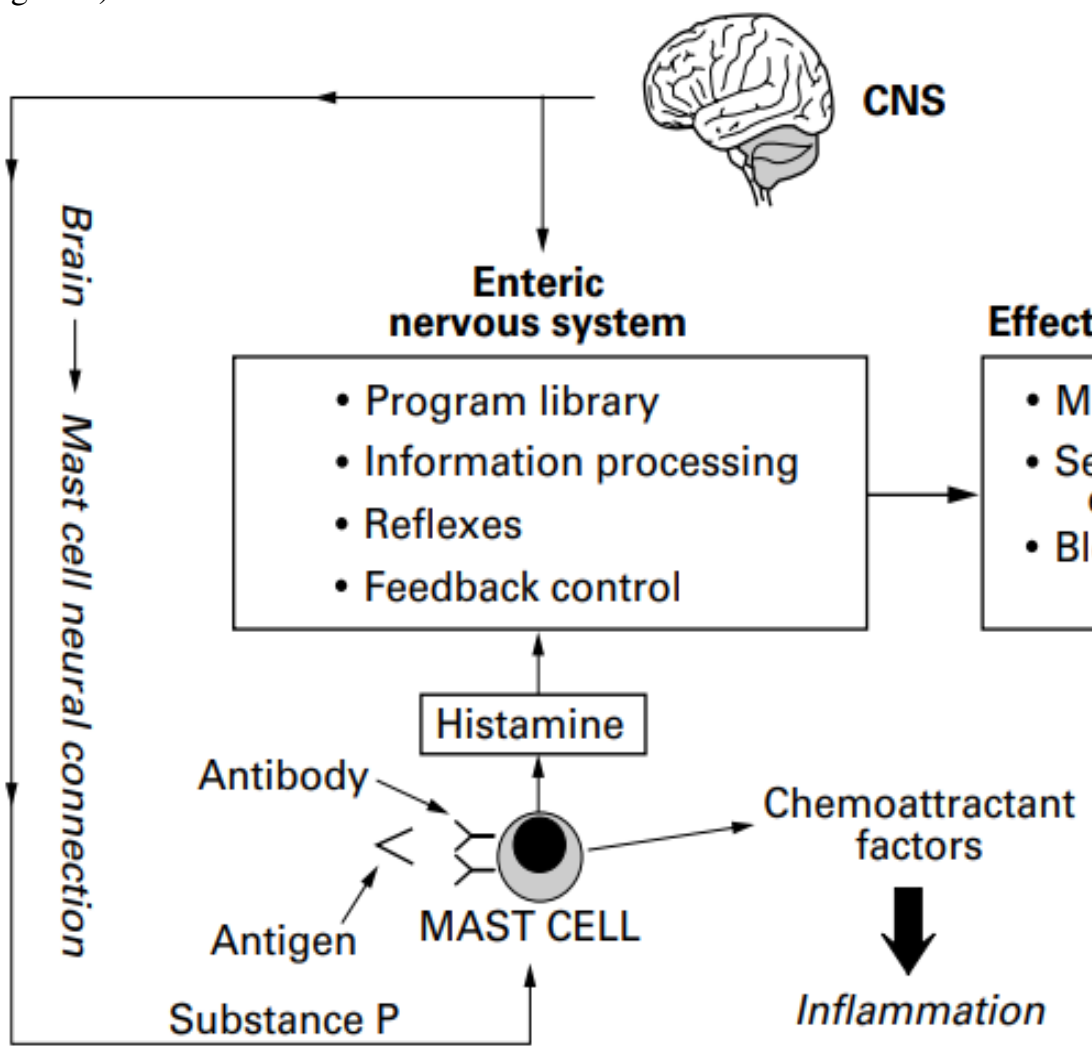

Effector systems

- Muscle

- Secretory epithelium

- Blood

\section{Behavior \\ $\left(\begin{array}{c}\text { Hyper-secretion } \\ \text { Power propulsion }\end{array}\right)$}

Symptoms

$\left(\begin{array}{c}\text { Diarrhea } \\ \text { pain }\end{array}\right)$

Figure 2. The relationship between the CNS, ENS and the functioning of the gut. The ENS is situated close to the gastrointestinal effectors it controls. Enteric mast cells signal the presence of foreign antigens to the ENS by releasing a number of paracrine mediators as part of an inflammatory response. The ENS then initiates propulsive motility that expels the source of antigenic stimulation from the bowel. This results in the clinical symptoms of abdominal pain and diarrhea. Impulses from the CNS to the mast cells have an effect on the ENS similar to that of an antigenicstimulation, resulting in similar symptoms (Reproduced from Wood et al. 1999).

Microbiota-gut-brain axis: a complex, interactive communication system

Although the ENS can function independent of the CNS, there is a constant interaction between the two systems through what is called the microbiota-gut-brain (MGB) axis. Broadly defined, the MGB-axis is a complex interplay between the gut microbiota, ENS, neuroendocrine, neuroimmune, parasympathetic and sympathetic systems and the CNS (Figure 3). This complex communication and interaction system results in the CNS being able to influence enteric 
behavior and, correspondingly, the gut being able to communicate with the brain (John Hopkins Medicine 2021). The CNS functions more as a receiver of impulses than a transmitter, as proved by the fact that about $90 \%$ of fibers of the vagus nerve (the $\mathrm{X}^{\text {th }}$ cranial nerve and the longest of the 12 cranial nerves) are those that carry impulses from the gut towards the brain (Forsythe et al. 2014). These impulses to the CNS, in turn, trigger off reflexes that regulate the motility of the GI tract (Furness et al. 2014).

The two-way interaction between the gut and the cognitive and emotional centers of the CNS has been substantiated by various studies. These include reports of mood changes arising from the transmission of impulses from the bowel to the CNS, association between various psychological disorders and GI tract symptoms referred to earlier (Privitera et al. 2013, Mussell et al. 2008, Severance et al. 2015, Edwards et al. 1993, Graff et al. 2009, Lim et al. 2014), and studies that have demonstrated improvement of learning, memory and depression by stimulating the vagus nerve ("vagal nerve stimulation', VNS). The vagus nerve is one the main components of the parasympathetic nervous system that controls the body's 'rest and digest' and 'feed and breed' responses, as opposed to the sympathetic nervous system that generates a 'fight or flight' response to impending danger. VNS modulates the transmission of impulses from the gut to the brain (Rush et al. 2000; George et al. 2000), and in the process also improves the functioning of certain cognitive and mood centres of the brain.

There also recent reports of 'bacteriotherapy' or fecal microbiota transplants (FMT) being able to improve symptoms in neurological disorders with a dysfunctional MGB axis (Vendrik et al. 2020). Studies have revealed promising effects of FMT in conditions such as ASD, multiple sclerosis, Parkinson's disease, epilepsy, stroke, Alzheimer's disease and GuillainBarré syndrome. In an interesting clinical trial, 18 children with ASD and GI tract symptoms demonstrated significant long-term improvement in their behavioural ASD symptoms 7 to 8 weeks after receiving daily doses of human gut microbiota with a drink or via enema (Kang et al. 2017).

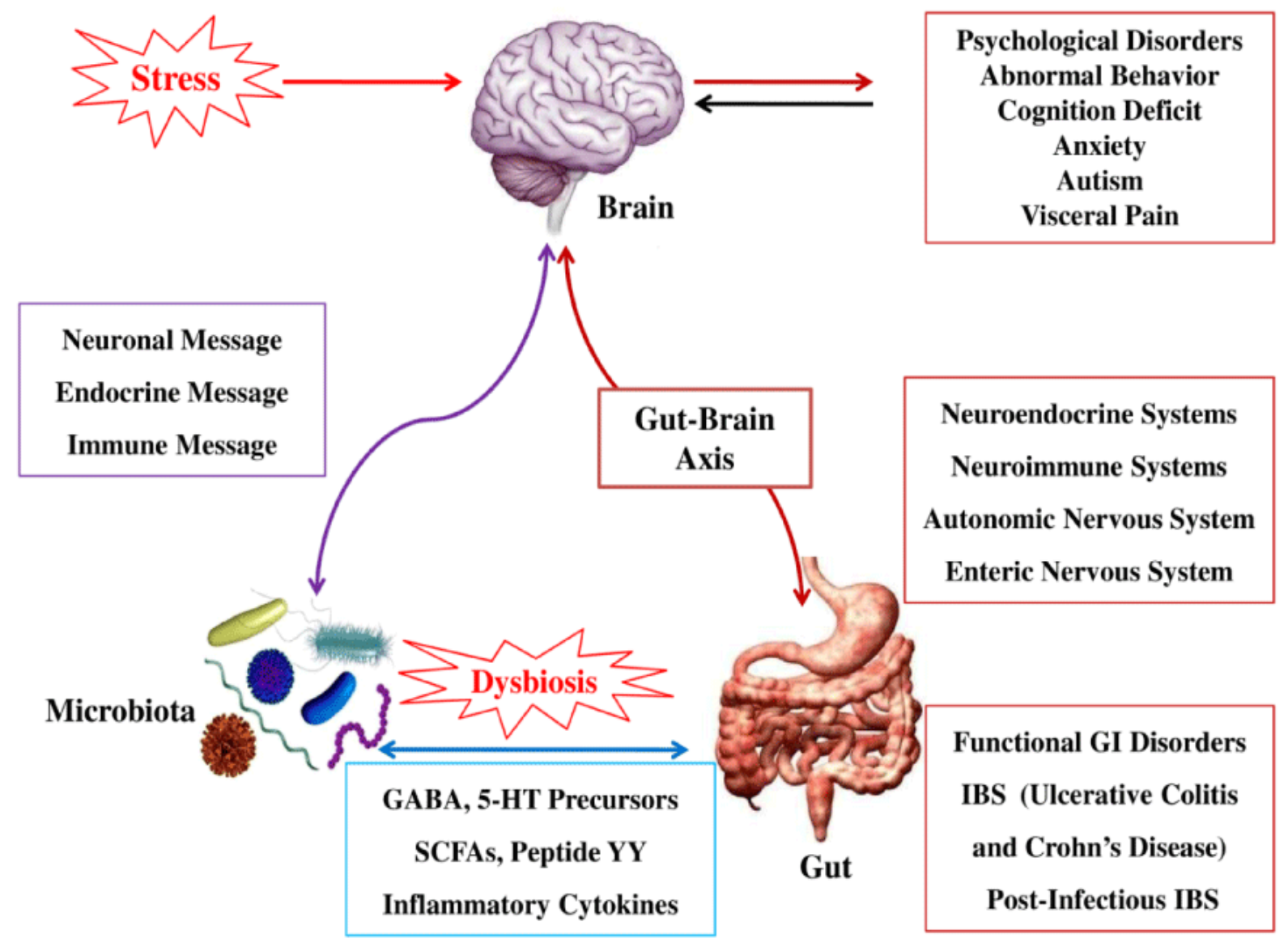


Figure 3. Microbiota-gut-brain axis. Pathways either directly or indirectly influence the bidirectional interactions between the gut microbiota and the CNS, involving endocrine, immune, and neural pathways (Reproduced from Thakur et al. 2014)

\section{Autism spectrum disorder}

The Autism spectrum disorder (ASD) is a gamut of neurodevelopmental disorders affecting 1 in 54 children in the United States. Comprising of autism, pervasive developmental disorders and Asperger's disorder, these disorders are broadly characterized by impairments in social interaction and communication and the presence of repetitive and stereotyped interests and behaviors (Johnson and Myers 2007). Though the American Psychiatric Association (APA) has laid down specific criteria for the diagnosis of ASD (American Psychiatric Association 2021), there is an overlap between the symptoms of ASD and non-ASD disorders. Interestingly, GI tract disturbances also figure in the broad scheme of these disorders (Figure 4).

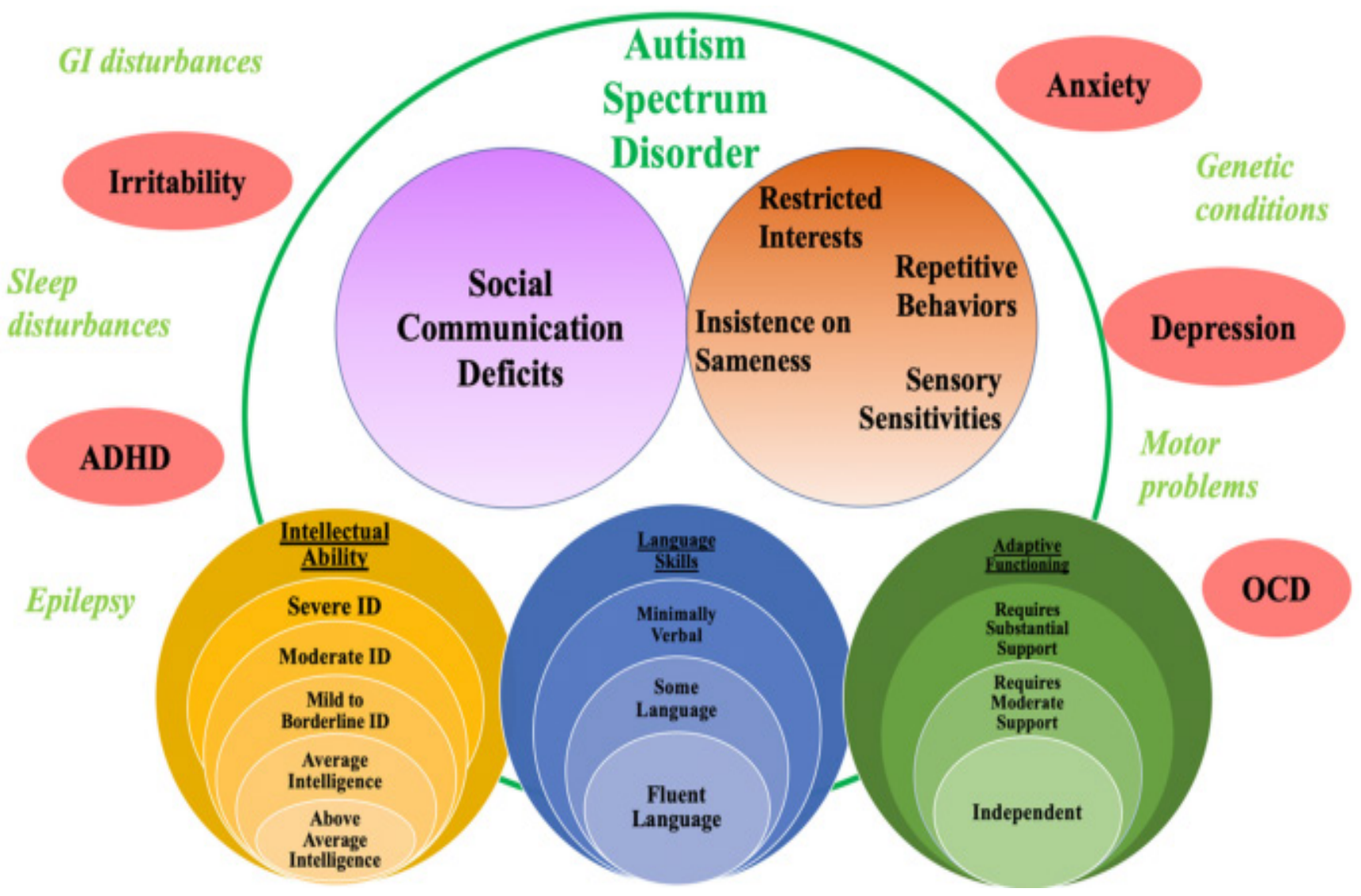

Figure 4. The symptom constellation in ASD. ADHD, attention deficit hyperactivity disorder; OCD, obsessive compulsive disorder (Reproduced from Rosen et al. 2021)

Ever since the disorder was first characterized, its diagnosis has been based on behavioral observations (Luyster et al. 2009) and criteria laid down by the APA (Klin et al. 2000) rather than any hard-core biomarkers. It is only recently that genetic markers of ASD have been identified by the process of 'reverse phenotyping', a technique that characterizes phenotypes based on particular genetic sequences (Benger et al. 2018). The genetic marker data so obtained has 
helped establish the role of genes such as the CHD8 and the 'psychiatric risk gene' transcription factor 4 (TCF4) in the pathogenesis of ASD.

Interestingly, research has revealed that variations in specific 'autism genes' also cause alterations in the MGB axis, indicating an underlying link between autism and the gut. This has been corroborated by experiments reporting the development of autism-like symptoms in germ-free mice, and reports of children with ASD having a distinct set of gut microbes and experiencing more GI tract symptoms compared to their non-ASD counterparts. A meta-analysis of 15 studies with a total of 2215 children (McElhanon et al. 2014) demonstrated that children with ASD were 2 to 4 times more likely than non-ASD children to experience abdominal pain, constipation and diarrhea. Evidence from such studies has spurred scientists on to decode the MGB-axis in ASD and explore the possibility of using this as a therapeutic target for a condition that currently has no approved medication.

\section{Microbiota-gut-brain axis in ASD}

\section{Intestinal barrier pathway: The 'leaky gut' hypothesis}

The epithelial integrity of the intestinal wall, also known as the 'intestinal barrier', is maintained by the metabolites of the gut microbiota. Defects in this barrier have been linked to factors such as altered gut microbiota, introduction of pathogenic bacteria, environment toxins and dietary macromolecules. These lead to a functionally impaired intestinal barrier with increased permeability, a condition known as 'leaky gut'. This 'leakiness' allows bacteria to cross over from the intestine into the blood and activate the secretion of cytokines by the brain, triggering an immune response (Figure 5) (Wu et al. 2015) that eventually affects specific cognitive functions of the brain.

Children with ASD have been demonstrated to have higher levels of immune-mediated inflammatory cytokines such as interleukins, tumor necrosis factor- $\alpha$ (TNF- $\alpha$ ) and transforming growth factor beta-1 (Masi et al. 2015) when compared to their non-ASD counterparts, leading to the postulate that their guts are 'leakier' than normal. Given that normal neural development in infants is altered by actions of these cytokines, it stands to reason that a stronger intestinal barrier would protect and positively impact their brain function. This explains why probiotics are being thoroughly investigated, both in the antenatal period and in children with ASD, for their possible role in mitigating some of the symptoms of ASD. There is also a potential underlying genetic basis for the cytokine variations seen in ASD (Liu et al. 2021), and this opens up prospects for exploring prenatal testing and targeted gene therapy for ASD in future.

\section{Neuronal Pathways}

Myelin is an insulating sheath that envelopes nerve cells and facilitates efficient transmission of electrical nerve impulses. Any alteration in the amount of myelin, and hence the thickness of the nerve sheath, can affect the transmission of nervous signals and result in the development of various neurological symptoms. Recent studies have found that a robust gut microbial colony is vital in regulating myelin-related genes in the prefrontal cortex of the brain- an area that is postulated to be affected in ASD. Experiments in germ-free mice axons demonstrated increased myelination in this region of the brain, a phenomenon that was reversed only on colonizing the brain with orthodox gut microbiota (Hoban et al. 2016). The results of this study support the association between healthy gut microbiota and a normally functioning prefrontal cortex. The clinical implication of this finding is that MGB axis dysfunction possibly correlates with the social dysfunction seen in ASD (Amaral et al. 2008).

The vagus nerve forms another vital neuronal pathway in the MGB-axis. It senses the microbiota metabolites and then transfers this information to the CNS to generate a specific response. Stress inhibits the vagus nerve and has deleterious effects on the gut microbiota and the GI tract. Targeting this nerve through procedures like VNS would thus be of interest to restore the balance in the MGB axis (Bonaz et al. 2018). In conventional VNS, a device is placed subcutaneously in the chest wall, and a wire from it is threaded to the left vagus nerve. When activated, the device sends electrical signals and 'stimulates' the vagus nerve. Such stimulation has been shown to have promising 
results in ASD. VNS in ASD patients with seizures not only reduced seizure frequency, but also resulted in an improvement in verbal skills, mood and alertness (Engineer et al. 2017).

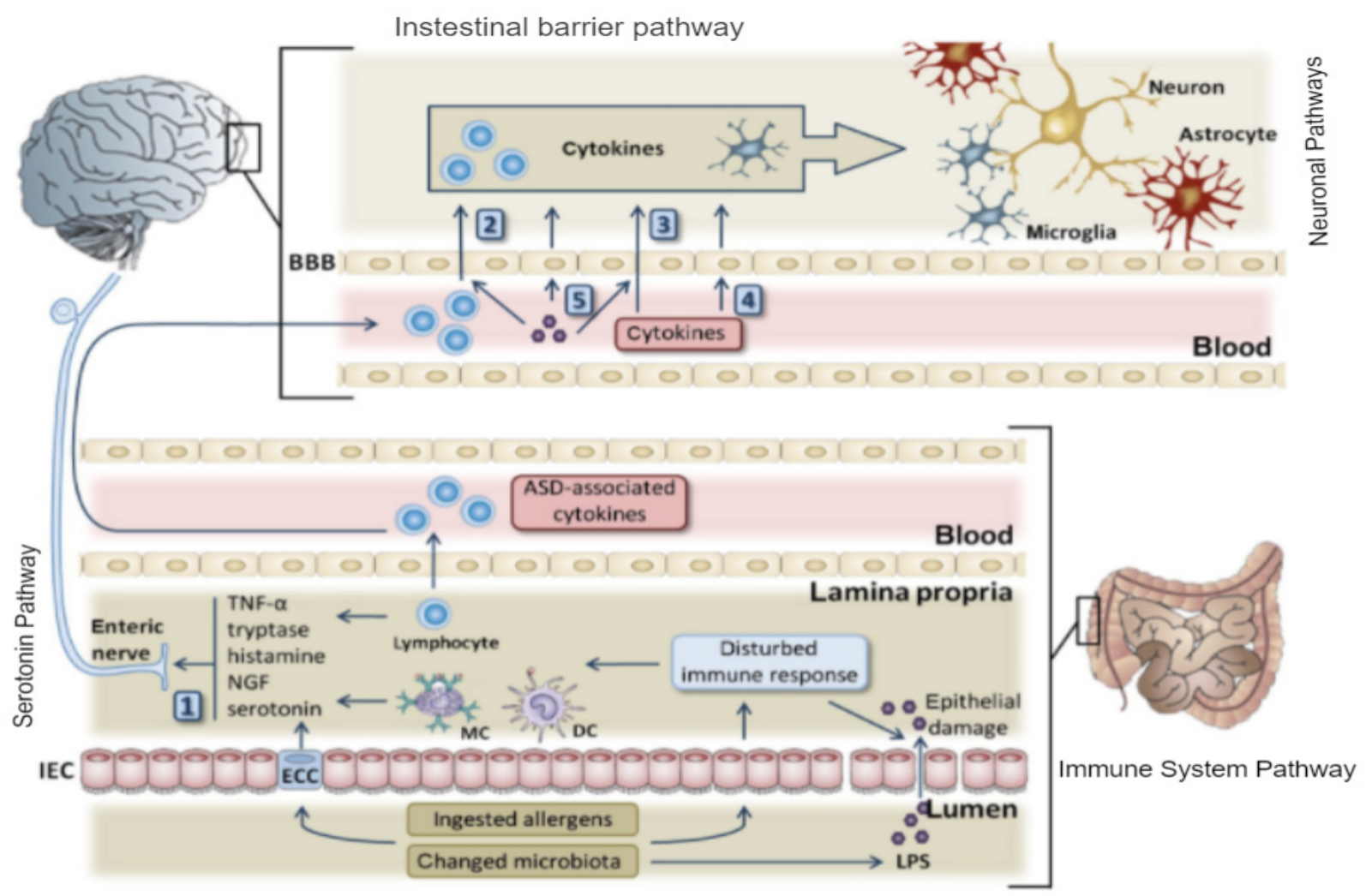

Figure 5. Different mechanisms implicating the microbiota-gut-brain axis in the pathogenesis of ASD. (Reproduced from Srikantha and Mohajeri 2019)

\section{The serotonin pathway}

The ENS utilizes more than 30 neurotransmitters (chemical messengers) for its normal functioning. Amongst these, serotonin, an important regulator of mood and cognition (Cryan and Leonard 2000), is postulated to be of significance in the MGB-axis in ASD. In addition to a well-defined role in adults, serotonin modulates neurodevelopment in children- specifically in the domains related to social function, repetitive behavior, and sensory development. Elevated levels of serotonin have been reported in children with ASD, suggesting the importance of serotonergic systems in the pathogenesis of ASD (Schain and Freedman 1961). Similar findings have been reported in adults with ASD. Serotonin availability is significantly lower in the CNS of adults with ASD compared with controls (Andersson et al. 2020). The findings of this study and many others endorse a significant association of serotonin with ASD. Since more than 90 percent of the body's serotonin is synthesized by the gut bacteria, and not in the CNS, it makes sense to target the MGB-axis to modify central serotonin levels which could then possibly alleviate the symptoms of ASD. Though the usage of selective serotonin reuptake inhibitors (SSRIs) currently has no evidence of benefit in children with ASD, serotonin still remains a promising heritable biomarker that could help identify individuals who may be more likely to benefit from future treatment protocols. 


\section{Immune system pathway}

ASD has been associated with a host of immunological abnormalities such as autoimmunity, activation of the 'immune-like' microglia and astroglia cells in the brain and increased T-cell activation (van Sadelhoff et al. 2019). Research over the past two decades suggests that pre-and post-natal immune dysregulation is a significant risk factor for ASD. Prenatal insults in the form of maternal infections may trigger immunological activation and increase the risk of ASD in the child. Postnatally, the affected children continue to demonstrate distinct profiles of immune dysregulation, inflammation, and endogenous autoantibodies (Meltzer and Van de Water 2017).

Besides the above systemic and CNS immunological imbalances, patients with ASD have also been found to harbor immune-related disturbances in their GI tract and the composition of the gut microbiota (Figure 5). It has also been suggested that specific amino-acids can strengthen the intestinal barrier, modify the mucosal immune system and target aberrant gut-CNS signaling pathways. It is hoped that such immunomodulation techniques can influence CNS neuronal activity and address the behavioral problems associated with ASD (van Sadelhoff et al. 2019)

\section{Metabolite pathway}

Metabolites such as short-chain fatty acids (SCFAs) produced by the gut bacteria have been demonstrated to be a source of energy for epithelial cells. The presence of such metabolites helps in the maintenance of the intestinal epithelial barrier and the important anti-inflammatory and immunological protection that it provides (Richards et al. 2016). Bacteria-derived SCFAs have also been recognized to be one of the key mediators of the gut-brain axis, and alterations in their production have been reported to occur in many neuro-psychological conditions.

In subjects with ASD, there is evidence from animal and epidemiological studies that SCFAs may be one of the environmental triggers for the condition (MacFabe 2015). Elevated levels of SCFAs have been reported in stool samples of children with ASD (Wang et al. 2012). In particular, propionic acid, a major SCFA produced by ASDassociated gut bacteria, has been shown to induce reversible ASD-like behavioral deficits in rats (Foley et al. 2015) It is postulated that the major effects of the SCFAs may occur through the alteration of mitochondrial function or by the modulation of specific ASD-genes, both of which may prove to be useful biomarkers and therapeutic targets in ASD.

\section{Conclusion}

The human gut houses a massive colony of microorganisms- the gut microbiota- that has been recognized to have far-reaching connections to the brain through a complex 'microbiota-gut-brain axis'. This axis has implications in the management of various neuro-psychological conditions including ASD. It is now widely recognized that ASD symptoms co-exist with GI tract symptoms and altered gut microbiota. The current study attempts to review the impact of the gut microbiota on the development of ASD, and elucidates how it could affect various pathways implicated in the disorder. The 'leaky gut' hypothesis suggests that the intestinal barrier in children with ASD is compromised, making them prone to the deleterious effects of cytokines. This indicates a promising therapeutic role of probiotics, both in the antenatal and postnatal periods, to alleviate the symptoms of ASD. The existence of neuronal pathways in the gut-brain axis implicates vagal nerve stimulation as a promising treatment modality for the disorder. The elucidation of serotonergic, immune-mediated and metabolite pathways opens up avenues for targeted therapies for ASD. Gut microbiota may thus hold the key to the treatment, and possibly even the prevention of ASD in future.

\section{Limitations}

Though the current study attempts to elucidate the commonly described pathways implicating an altered MCB-axis in ASD, it is not an exhaustive review on the intricacies of the topic. Each of the described pathways has finer biological substrates and connotations that need further research and clarity. Genetic aberrations described in relation to some of the proposed pathways, in addition to other under-explored biomarkers, may provide a more wholesome understanding of ASD. 


\section{Acknowledgments}

I would like to thank Avan Erhunmwunsee Dalton, Department of Biomedical Engineering, College of Medicine, University of Lagos, Nigeria for guiding me in researching about this topic and writing up the paper. I sincerely appreciate his unique insights in this regard.

\section{References}

Amaral, D. G., Schumann, C. M., \& Nordahl, C. W. (2008). Neuroanatomy of autism. Trends in neurosciences, 31(3), 137-145. https://doi.org/10.1016/j.tins.2007.12.005

American Psychiatric Association, What Is Autism Spectrum Disorder? Accessed May 30, 2021 https://www.psychiatry.org/patients-families/autism/what-is-autism-spectrum-disorder

Andersson, M., Tangen, Ä., Farde, L., Bölte, S., Halldin, C., Borg, J., \& Lundberg, J. (2020). Serotonin transporter availability in adults with autism-a positron emission tomography study. Molecular Psychiatry, 1-12. https://doi.org/10.1038/s41380-020-00868-3

Benger, M., Kinali, M., \& Mazarakis, N. D. (2018). Autism spectrum disorder: prospects for treatment using gene therapy. Molecular autism, 9(1), 1-10. https://doi.org/10.1186/s13229-018-0222-8

Bonaz, B., Bazin, T., \& Pellissier, S. (2018). The vagus nerve at the interface of the microbiota-Gut-Brain Axis. Front. Neurosci. Frontiers Media SA. https://doi.org/10.3389/fnins.2018.00049

Crumeyrolle-Arias, M., Jaglin, M., Bruneau, A., Vancassel, S., Cardona, A., Daugé, V., ... \& Rabot, S. (2014). Absence of the gut microbiota enhances anxiety-like behavior and neuroendocrine response to acute stress in rats. Psychoneuroendocrinology, 42, 207-217. https://doi.org/10.1016/j.psyneuen.2014.01.014

Cryan, J. F., \& Leonard, B. E. (2000). 5-HT1A and beyond: the role of serotonin and its receptors in depression and the antidepressant response. Human Psychopharmacology: Clinical and Experimental, 15(2), 113-135. https://doi.org/10.1002/(SICI)1099-1077(200003)15:2<113::AID-HUP150>3.0.CO;2-W

Edwards, L. L., Quigley, E. M. M., Hofman, R., \& Pfeiffer, R. F. (1993). Gastrointestinal symptoms in Parkinson disease: 18-month follow-up study. Movement disorders: official journal of the Movement Disorder Society, 8(1), 8386. https://doi.org/10.1002/mds.870080115

Engineer, C. T., Hays, S. A., \& Kilgard, M. P. (2017). Vagus nerve stimulation as a potential adjuvant to behavioral therapy for autism and other neurodevelopmental disorders. Journal of neurodevelopmental disorders, 9(1), 1-8. https://doi.org/10.1186/s11689-017-9203-z

Foley, K. A., MacFabe, D. F., Vaz, A., Ossenkopp, K. P., \& Kavaliers, M. (2014). Sexually dimorphic effects of prenatal exposure to propionic acid and lipopolysaccharide on social behavior in neonatal, adolescent, and adult rats: implications for autism spectrum disorders. International Journal of Developmental Neuroscience, 39, 68-78. https://doi.org/10.1016/j.ijdevneu.2014.04.001

Forsythe, P., Bienenstock, J., \& Kunze, W. A. (2014). Vagal pathways for microbiome-brain-gut axis communication. Microbial endocrinology: the microbiota-gut-brain axis in health and disease, 115-133. https://doi.org/10.1007/978-1-4939-0897-4_5

Furness, J. B. (2006). The enteric nervous system Blackwell Publishing. Melbourne, Australia.

Furness, J. B., Callaghan, B. P., Rivera, L. R., \& Cho, H. J. (2014). The enteric nervous system and gastrointestinal innervation: integrated local and central control. Microbial endocrinology: The microbiota-gut-brain axis in health and disease, 39-71. https://doi.org/10.1007/978-1-4939-0897-4_3

George, M. S., Sackeim, H. A., Rush, A. J., Marangell, L. B., Nahas, Z., Husain, M. M., ... \& Ballenger, J. C. (2000). Vagus nerve stimulation: a new tool for brain research and therapy. Biological psychiatry, 47(4), $287-295$. https://doi.org/10.1016/S0006-3223(99)00308-X 
Graff, L. A., Walker, J. R., \& Bernstein, C. N. (2009). Depression and anxiety in inflammatory bowel disease: a review of comorbidity and management. Inflammatory bowel diseases, 15(7), 1105-1118. https://doi.org/10.1002/ibd.20873 Hoban, A. E., Stilling, R. M., Ryan, F. J., Shanahan, F., Dinan, T. G., Claesson, M. J., ... \& Cryan, J. F. (2016). Regulation of prefrontal cortex myelination by the microbiota. Translational psychiatry, 6(4), e774-e774. https://doi.org/10.1038/tp.2016.42

John Hopkins Medicine. The Brain-Gut Connection. Accessed May 30, 2021 https://www.hopkinsmedicine.org/health/wellness-and-prevention/the-brain-gut-connection

Johnson, C. P., \& Myers, S. M. (2007). Identification and evaluation of children with autism spectrum disorders. Pediatrics, 120(5), 1183-1215. https://doi.org/10.1542/peds.2007-2361

Kang, D.W., Adams, J.B., Gregory, A.C., Borody, T., Chittick, L., Fasano, A., ... \& Krajmalnik-Brown R. (2017) Microbiota Transfer Therapy alters gut ecosystem and improves gastrointestinal and autism symptoms: an open-label study. Microbiome, 23;5(1),10. https://doi.org/10.1186/s40168-016-0225-7

Klin, A., Lang, J., Cicchetti, D. V., \& Volkmar, F. R. (2000). Brief report: Interrater reliability of clinical diagnosis and DSM-IV criteria for autistic disorder: Results of the DSM-IV autism field trial. Journal of autism and Developmental disorders, 30(2), 163. 10.1023/A:1005415823867

Langley, J. N. (2010). The Autonomic Nervous System, Part 1 [1921] Cornell Univ.

Lim, W. Y., Subramaniam, M., Abdin, E., Vaingankar, J., \& Chong, S. A. (2014). Peptic ulcer disease and mental illnesses. General hospital psychiatry, 36(1), 63-67. https://doi.org/10.1016/j.genhosppsych.2013.09.004

Liu, Z., Mao, X., Dan, Z., Pei, Y., Xu, R., Guo, M., ...\& Liu, X. (2021) Gene variations in autism spectrum disorder are associated with alteration of gut microbiota, metabolites and cytokines. Gut Microbes,13(1),1-16. https://doi.org/10.1080/19490976.2020.1854967

Luyster, R., Gotham, K., Guthrie, W., Coffing, M., Petrak, R., Pierce, K., ... \& Lord, C. (2009). The Autism Diagnostic Observation Schedule-Toddler Module: A new module of a standardized diagnostic measure for autism spectrum disorders. Journal of autism and developmental disorders, 39(9), 1305-1320. https://doi.org/10.1007/s10803-0090746-Z

MacFabe, D. F. (2015). Enteric short-chain fatty acids: microbial messengers of metabolism, mitochondria, and mind: implications in autism spectrum disorders. Microbial ecology in health and disease, $26(1), 28177$. 10.3402/mehd.v26.28177

Masi, A., Quintana, D. S., Glozier, N., Lloyd, A. R., Hickie, I. B., \& Guastella, A. J. (2015). Cytokine aberrations in autism spectrum disorder: a systematic review and meta-analysis. Molecular psychiatry, 20(4), $440-446$. https://doi.org/10.1038/mp.2014.59

McElhanon, B. O., McCracken, C., Karpen, S., \& Sharp, W. G. (2014). Gastrointestinal symptoms in autism spectrum disorder: a meta-analysis. Pediatrics, 133(5), 872-883. https://doi.org/10.1542/peds.2013-3995

Meltzer, A., \& Van de Water, J. (2017). The role of the immune system in autism spectrum disorder. Neuropsychopharmacology, 42(1), 284-298. https://doi.org/10.1038/npp.2016.158

Mussell, M., Kroenke, K., Spitzer, R. L., Williams, J. B., Herzog, W., \& Löwe, B. (2008). Gastrointestinal symptoms in primary care: prevalence and association with depression and anxiety. Journal of psychosomatic research, 64(6), 605-612. https://doi.org/10.1016/j.jpsychores.2008.02.019

Privitera, G. J., Misenheimer, M. L., \& Doraiswamy, P. M. (2013). From weight loss to weight gain: appetite changes in major depressive disorder as a mirror into brain-environment interactions. Frontiers in psychology, $4,873$. https://doi.org/10.3389/fpsyg.2013.00873

Rao, M., \& Gershon, M. D. (2016). The bowel and beyond: the enteric nervous system in neurological disorders. Nature reviews Gastroenterology \& hepatology, 13(9), 517. https://doi.org/10.1038/nrgastro.2016.107

Richards, J. L., Yap, Y. A., McLeod, K. H., Mackay, C. R., \& Mariño, E. (2016). Dietary metabolites and the gut microbiota: an alternative approach to control inflammatory and autoimmune diseases. Clinical \& translational immunology, 5(5), e82. https://doi.org/10.1038/cti.2016.29 
Rosen, N. E., Lord, C., \& Volkmar, F. R. (2021). The Diagnosis of Autism: From Kanner to DSM-III to DSM-5 and Beyond. Journal of Autism and Developmental Disorders, 1-18. https://doi.org/10.1007/s10803-021-04904-1

Rush, A. J., George, M. S., Sackeim, H. A., Marangell, L. B., Husain, M. M., Giller, C., ... \& Goodman, R. (2000). Vagus nerve stimulation (VNS) for treatment-resistant depressions: a multicenter study. Biological psychiatry, 47(4), 276-286. https://doi.org/10.1016/S0006-3223(99)00304-2

Schain, R. J., \& Freedman, D. X. (1961). Studies on 5-hydroxyindole metabolism in autistic and other mentally retarded children. The Journal of pediatrics, 58(3), 315-320. https://doi.org/10.1016/S0022-3476(61)80261-8

Sender, R., Fuchs, S., \& Milo, R. (2016). Revised estimates for the number of human and bacteria cells in the body. PLoS biology, 14(8), e1002533. https://doi.org/10.1371/journal.pbio.1002533

Severance, E. G., Prandovszky, E., Castiglione, J., \& Yolken, R. H. (2015). Gastroenterology issues in schizophrenia: why the gut matters. Current psychiatry reports, 17(5), 27. https://doi.org/10.1007/s11920-015-0574-0

Srikantha, P., \& Mohajeri, M. H. (2019). The possible role of the microbiota-gut-brain-axis in autism spectrum disorder. International Journal of molecular sciences, 20(9), 2115. https://doi.org/10.3390/ijms20092115

Thakur, A. K., Shakya, A., Husain, G. M., Emerald, M., \& Kumar, V. (2014). Gut-microbiota and mental health: current and future perspectives. J Pharmacol Clin Toxicol, 2(1), 1016.

van Sadelhoff, J. H., Perez Pardo, P., Wu, J., Garssen, J., Van Bergenhenegouwen, J., Hogenkamp, A., ... \& Kraneveld, A. D. (2019). The gut-immune-brain axis in autism spectrum disorders; a focus on amino acids. Frontiers in endocrinology, 10, 247. https://doi.org/10.3389/fendo.2019.00247

Vendrik, K. E., Ooijevaar, R. E., de Jong, P. R., Laman, J. D., van Oosten, B. W., van Hilten, J. J., ... \& Contarino, M. F. (2020). Fecal microbiota transplantation in neurological disorders. Frontiers in cellular and infection microbiology, 10, 98. https://doi.org/10.3389/fcimb.2020.00098

Wang, L., Christophersen, C. T., Sorich, M. J., Gerber, J. P., Angley, M. T., \& Conlon, M. A. (2012). Elevated fecal short chain fatty acid and ammonia concentrations in children with autism spectrum disorder. Digestive diseases and sciences, 57(8), 2096-2102. https://doi.org/10.1007/s10620-012-2167-7

Wood, J. D., Alpers, D. H., \& Andrews, P. L. R. (1999). Fundamentals of neurogastroenterology. Gut, 45(suppl 2), II6-II16. http://dx.doi.org/10.1136/gut.45.2008.ii6

Wu, S., Yi, J., Zhang, Y. G., Zhou, J., \& Sun, J. (2015). Leaky intestine and impaired microbiome in an amyotrophic lateral sclerosis mouse model. Physiological reports, 3(4). https://doi.org/10.14814/phy2.12356 